\title{
A practical approach to the cholesterol guidelines and ASCVD prevention
}

\section{ABSTRACT}

Lifestyle factors remain the bedrock of atherosclerotic cardiovascular disease (ASCVD) prevention. Statins remain the first-line therapy for primary and secondary prevention of ASCVD. Physicians are encouraged to discuss the risks and benefits of statins with patients before beginning therapy. Nonstatins and proprotein convertase subtilisin-kexin type 9 inhibitors are recommended for secondary prevention in patients with clinical ASCVD. For patients at intermediate risk of ASCVD, new risk-enhancing factors, including chronic inflammatory conditions and ethnicity, should be considered to better risk stratify these patients.

\section{KEY POINTS}

Lifestyle factors are the cornerstone of preventing ASCVD.

High low-density lipoprotein cholesterol (LDL-C) levels warrant intervention.

Statins are the first-line therapy for lowering LDL-C and preventing ASCVD.

Calcium score and risk-enhancing factors help stratify risk in patients of intermediate risk.

Nonstatin agents are warranted in patients not achieving LDL-C reduction goals despite maximum statin therapy or in primary prevention for patients at very-high risk and not achieving LDL-C reduction goals.

Dr. Cho reported research trial support from Amgen, Novartis, and Esperion and consulting/advisory fees from Amgen, Esperion, and AstraZeneca.

This article is based on Dr. Cho's presentation at the Sones/Favaloro Scientific Program, "Multidisciplinary Management of Acute and Chronic Conditions: Yielding Exceptional Outcomes," held in Cleveland, OH, November 8, 2019. The article was drafted by Cleveland Clinic Journal of Medicine and was then reviewed, revised, and approved by Dr. Cho.

doi:10.3949/ccjm.87.s1.02

\section{INTRODUCTION}

Maintaining healthy cholesterol levels is essential to primary and secondary prevention of atherosclerotic cardiovascular disease (ASCVD). High cholesterol raises the risk of heart disease, which is the leading cause of death in the United States. ${ }^{1}$ Just over $50 \%$ of US adults who would benefit from cholesterollowering medication are taking it. ${ }^{2}$

\section{CHOLESTEROL GUIDELINES}

The recent 2018 cholesterol guidelines for prevention of ASCVD again stress the importance of lifestyle and role of statins as first-line therapy in primary and secondary prevention. The guidelines affirm the need to aggressively treat patients with high cholesterol and particularly patients at high risk of ASCVD.

Table 1 highlights key updates to the cholesterol guidelines with respect to a clinician-patient risk discussion before beginning statins, tailoring treatment using a more extensive list or risk modifiers, additional testing for patients of intermediate risk, and use of nonstatin agents in secondary prevention.,

\section{WHAT REMAINS THE SAME}

Like the 2013 cholesterol guidelines, the new 2018 guidelines stress lifestyle, such as diet and exercise, statin therapy as the first-line drug for treating for atherosclerosis risk reduction, and risk assessment using the Pooled Cohort Equation in primary prevention of ASCVD.

\section{Lifestyle: Cornerstone of ASCVD prevention}

Lifestyle factors such as tobacco cessation, diet, exercise, weight management, and blood pressure management remain the cornerstone of primary and secondary prevention of ASCVD. A healthy diet that ensures the best nutritional value for caloric intake is recommended, such as a Mediterranean-style diet including whole grains and limiting red meat.

Unfortunately, the reality is that the lifestyle of very few Americans meets the lifestyle factor guide- 
TABLE 1

Key updates (in bold) to the cholesterol guidelines

\begin{tabular}{|c|c|c|}
\hline Topic & 2013 & 2018 \\
\hline Lifestyle & Cornerstone of ASCVD and prevention & Cornerstone of ASCVD and prevention \\
\hline First-line drugs for ASCVD risk reduction & Statins & Statins \\
\hline Clinician-patient risk discussion & Introduced & Emphasized before beginning statin \\
\hline Risk assessment in primary prevention & Starts with PCE risk estimation & Starts with PCE risk estimation \\
\hline Tailoring treatment & Some biomarkers and noninvasive tests & Extensive list of risk enhancers \\
\hline Intermediate risk patients & No recommendation for additional testing & Consider CAC scoring and risk enhancers \\
\hline $\begin{array}{l}\text { Nonstatin agents in secondary } \\
\text { prevention }\end{array}$ & None & $\begin{array}{l}\text { Ezetimib (Zetia) } \\
\text { PCSK9 inhibitors }\end{array}$ \\
\hline
\end{tabular}

ASCVD = atherosclerotic cardiovascular disease; $C A C=$ coronary artery calcium; PCE = Pooled Cohort Equation; PCSK9 = proprotein convertase subtilisin-kexin type 9

Source: Data from references 3 and 4.

lines. The average American gets about 16 grams of fiber a day ${ }^{5}$ as opposed to the recommended 30 grams. ${ }^{6}$ Total animal consumption per capita in the United States has steadily increased and currently stands at 225 pounds of meat. ${ }^{7}$

For exercise, the American Heart Association recommends 30 minutes or more of moderate-intensity aerobic activity at least 5 days a week and high-intensity strengthening at least 2 days a week. ${ }^{8}$ As with diet, Americans fall short of exercise recommendations with $39 \%$ considered sedentary, $60 \%$ get no regular exercise, and of those who exercise, $80 \%$ are not exercising effectively. Rates of sedentary lifestyle also increase with age. ${ }^{9}$

These data are clear that most patients do not meet lifestyle recommendations for diet and exercise to prevent ASCVD.

\section{Patient factors that warrant treatment}

As stated, adherence to a healthy lifestyle is the primary prevention of ASCVD for all patients (Figure 1). Treatment to maintain healthy cholesterol and prevent cardiovascular disease is warranted in patients who have had clinical ASCVD (Figure 2); for everyone else, treatment is based on a calculation of risk, including other conditions such as hypercholesterolemia and diabetes:

- Primary hypercholesterolemia (low-density lipoprotein cholesterol [LDL-C] > $190 \mathrm{mg} / \mathrm{dL}$ )

- Diabetes and age 40 to 75

- Others factors based on calculated risk (Pooled Cohort Equation, coronary artery calcium [CAC], risk-enhancers)

- ASCVD including myocardial infraction, coronary artery disease, percutaneous coronary intervention/coronary artery bypass grafting, other arterial revascularization, transient ischemic attack, and stroke.

High LDL-C warrants statin therapy. In addition to unfavorable lifestyle factors, high LDL-C is a known risk factor for ASCVD and it warrants intervention. Regardless of age, patients with LDL-C greater than $190 \mathrm{mg} / \mathrm{dL}$ should be started on a statin therapy. The latest guidelines now encourage a discussion of ASCVD risk between the clinician and patient before beginning statin therapy. ${ }^{4}$

The selection and use of statin therapy is based on the LDL-C reduction goals as discussed below.

Calculated risk. In the absence of prior ASCVD, determining a patient's 10-year risk of ASCVD starts with a risk calculation such as the Pooled Cohort Equation. Risk calculation is based on age, sex, race, blood pressure, cholesterol, tobacco use, history of diabetes, and treatment with a statin or aspirin or for hypertension. A result of $5 \%$ to $7.4 \%$ indicates moderate 10-year risk of ASCVD and 7.5\% or greater indicates a high 10-year risk (http://tools. acc.org/ASCVD-Risk-Estimator-Plus/\#!/calculate/ estimate/).

For patients with a calculated risk of ASCVD in the moderate range, the new guidelines contain several new important risk enhancers to consider, as discussed below.

\section{WHAT IS NEW}

In addition to a patient-physician discussion before beginning statins, the biggest changes in the new 
Emphasize adherence to healthy lifestyle and assess ASCVD risk in each age group.

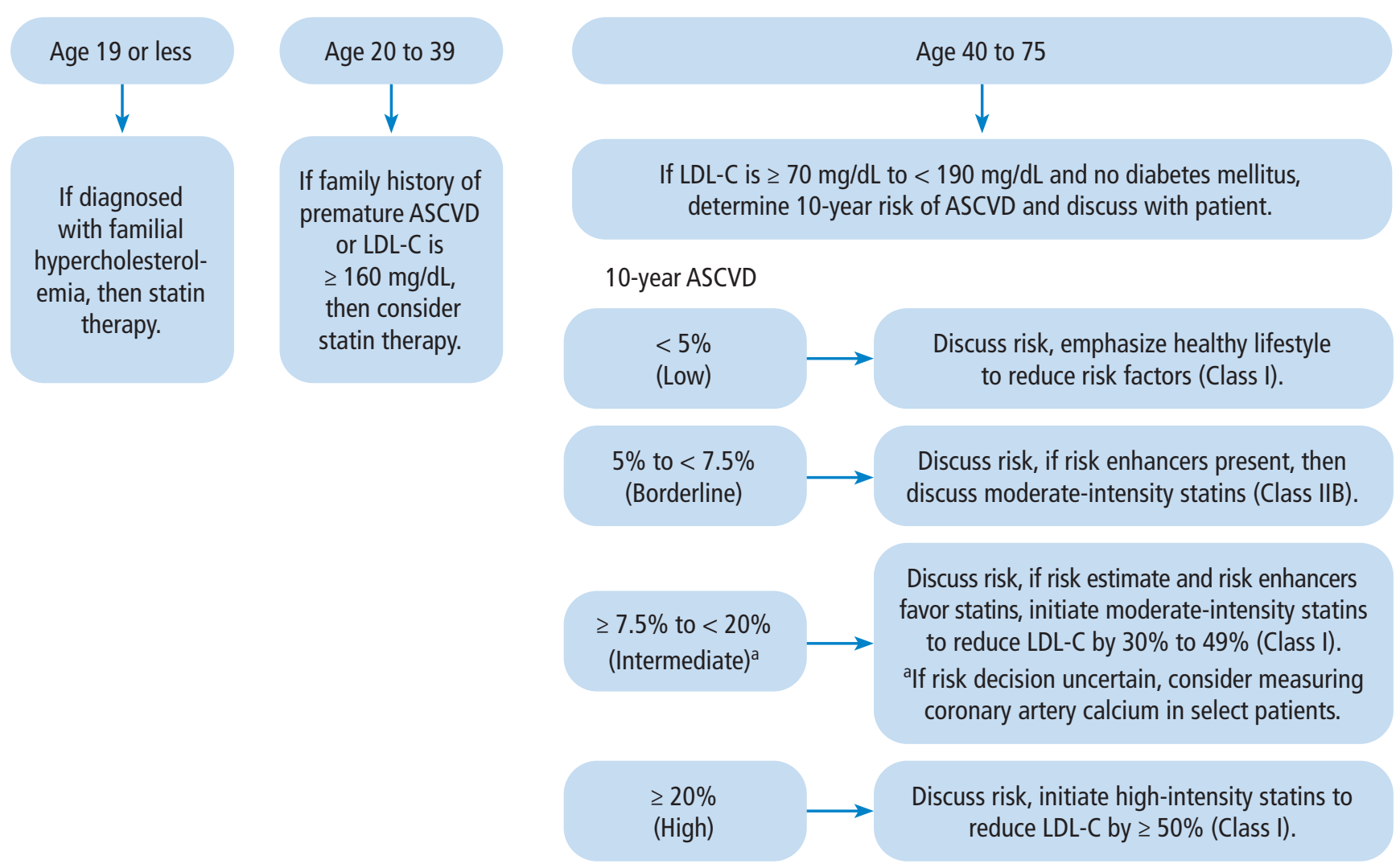

Figure 1. Primary prevention for atherosclerotic cardiovascular disease (ASCVD).

LDL-C = low-density lipoprotein cholesterol

Source: Data from reference 4.

cholesterol guidelines relate to identifying patients at intermediate risk of ASCVD and how best to stratify their level of risk.

\section{Intermediate risk}

The 2018 guidelines recommend new tools to further stratify patients at intermediate risk for ASCVD using CAC scoring and other new risk-enhancing factors.

CAC scoring. CAC score measures the amount of calcium in the artery walls of the heart and arterial plaque and can help further stratify patients with an intermediate risk for ASCVD. For patients at moderate risk and unsure about use of statin therapy, a CAC score may help inform risk-benefit treatment decisions. A CAC score of:

- A 0 indicates lower risk and favors continued dietary and lifestyle modifications and no statin therapy unless diabetes, family history of prema- ture coronary heart disease, or cigarette smoking are present

- 1 to 99 indicates elevated risk and favors use of statin therapy especially after age 55

- 100 or greater or the 75 th percentile or greater, indicates the need to initiate statin therapy.

Risk-enhancing factors. The latest cholesterol guidelines include an extensive list of risk-enhancing factors (Table 2). Family history is a risk factor, as in the past, in addition to metabolic disease, primary hypercholesterolemia, and chronic kidney disease.

Notably, chronic inflammatory conditions, such as psoriasis, rheumatoid arthritis, and lupus, are now considered risk-enhancing factors. Research indicates that autoimmune disease increases the risk for atherosclerosis by $300 \%$ to $500 \% .^{10}$

The risk factors related to autoimmune disease are particularly relevant to women because 


\section{Clinical ASCVD}

Adhere to healthy lifestyle and assess ASCVD risk in each age group
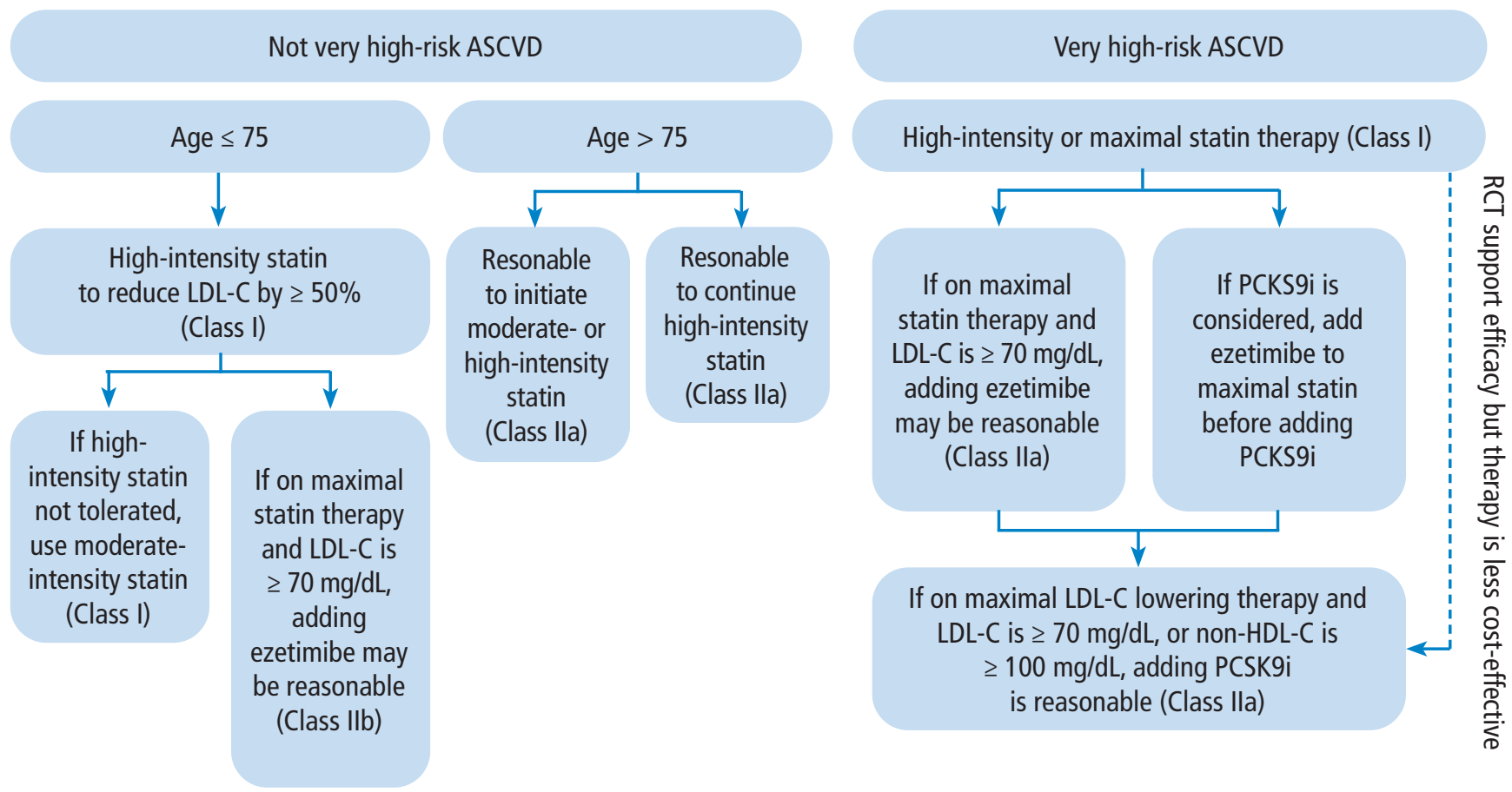

Figure 2. Secondary prevention for atherosclerotic cardiovascular disease (ASCVD).

HDL-C = high-density lipoprotein cholesterol; LDL-C = low-density lipoprotein cholesterol; PCKS9i = proprotein convertase subtilisin-kexin type 9 inhibitors; $\mathrm{RCT}=$ randomized controlled trial

Source: Data from reference 4 .

$80 \%$ of autoimmune disease occurs in women. Additional risk enhancers unique to women are premature menopause, menopause before the age of 40 regardless of whether surgically or naturally, preeclampsia, gestational diabetes, and polycystic ovary syndrome.

Another novel factor included in the new guidelines is high-risk ethnicity among South Asians. Lipid and biomarker factors include high-sensitive C-reactive protein, lipoprotein(a), apolipoprotein B, and ankle-brachial index. Lipoprotein(a) is a genetic disorder occurring in $20 \%$ of the population and it increases the risk of early $\mathrm{MI}$ and stroke. ${ }^{11} \mathrm{~A}$ randomized, phase 3 trial in 8,000 patients of an antisense oligonucleotide drug for cardiovascular disease and lipoprotein(a) is underway (NCT04023552 available at clinicaltrials.gov).

The new extensive list of risk enhancers should be considered to risk stratify and tailor treatment in patients with an intermediate risk of ASCVD.

\section{Very high risk/secondary prevention: Nonstatins and PCSK9 inhibitors}

Finally, there is new guidance about the role of nonstatin agents, such as ezetimibe and proprotein convertase subtilisin-kexin type 9 (PCSK9) inhibitors, in secondary prevention. Very high risk of ASCVD exists in patients with a clinical history of a major atherosclerotic event, recent ASCVD, myocardial infarction, or ischemic stroke. These high-risk patients should have an LDL-C less than $70 \mathrm{mg} / \mathrm{dL}$, though the Endocrine Society recommends LDL-C less than $55 \mathrm{mg} / \mathrm{dL}$ in patients diabetes and high-risk features for ASCVD. ${ }^{12}$

There are 3 nonstatin drugs currently available for LDL-C reduction (Table 3). Ezetimibe is a cholesterol absorption inhibitor shown to lower LDL-C by $18 \%$ taken as monotherapy and 25\% taken as combination therapy. ${ }^{4}$

The 2 available PCSK9 inhibitors, alirocumab and evolocumab, inhibit LDL-C receptors from breaking 
TABLE 2

\section{Risk-enhancing factors for ASCVD}

\begin{tabular}{ll}
\hline Factor & Findings \\
\hline Family history premature & Males: age $<55$ \\
ASCVD & Females: age $<65$ \\
Primary & LDL-C: $160-189 \mathrm{mg} / \mathrm{dL}$ \\
hypercholesterolemia & $(4.1-4.8 \mathrm{mmol} / \mathrm{L})$ \\
& Non-HDL-C: $190-219 \mathrm{mg} / \mathrm{dL}$ \\
& $(4.9-5.6 \mathrm{mmol} / \mathrm{L})$ \\
Metabolic syndrome & Waist circumference: increased \\
(3 or more) & Triglycerides: $>175 \mathrm{mg} / \mathrm{dL}$ \\
& Low HDL-C: $<40 \mathrm{mg} / \mathrm{dL}$, men; \\
& $<50 \mathrm{mg} / \mathrm{dL}$, women \\
& Elevated blood pressure \\
& Elevated glucose \\
Specific to women & Premature menopause \\
& (before 40$)$ \\
& Pre-eclampsia \\
Chronic kidney disease & eGFR: $15-59 \mathrm{~mL} / \mathrm{min} / 1.73 \mathrm{~m}{ }^{2}$ \\
& with or without albuminuria \\
& Not treated with dialysis \\
& or transplant \\
Chronic inflammatory & Psoriasis, rheumatoid arthritis, \\
conditions & HIV/AIDS \\
High-risk ethnicity & South Asian \\
Lipids/biomarkers & hs-CRP: $\geq 2.0 \mathrm{mg} / \mathrm{L}$ \\
& Lp(a): $\geq 50 \mathrm{mg} / \mathrm{dL}(\geq 125 \mathrm{nmol} / \mathrm{L}$ ) \\
& apoB: $\geq 130 \mathrm{mg} / \mathrm{dL}$ \\
& ABI: $<0.9$ \\
& \\
\hline
\end{tabular}

$\mathrm{ABI}=$ ankle-brachial index $; \mathrm{apoB}=$ apolipoprotein $\mathrm{B} ; \mathrm{ASCVD}=$ atherosclerotic cardiovascular disease; eGFR = estimated glomerular filtration rate; $\mathrm{HDL}-\mathrm{C}=$ high-density lipoprotein cholesterol; hs-CRP = high-sensitive C-reactive protein; LDL-C = low-density lipoprotein cholesterol; $\operatorname{Lp}(\mathrm{a})=$ lipoprotein(a)

down. ${ }^{4}$ Reduction of LDL-C is reportedly similar at $45 \%$ to $58 \%$ for alirocumab and $58 \%$ to $64 \%$ evolocumab depending on the dose. ${ }^{4}$

A very interesting finding about PCSK9 inhibitors is that unlike statins, no matter how low LDL-C levels go, there is no increased in the risk of diabetes. ${ }^{13}$
TABLE 3

Nonstatin therapy for reduction of LDL-C

\begin{tabular}{|c|c|c|}
\hline Drug & Dose & $\begin{array}{l}\text { Average LDL-C } \\
\text { reduction }\end{array}$ \\
\hline Ezetimibe & 10 mg, daily & $\begin{array}{l}18 \% \text { monotherapy } \\
25 \% \text { combined } \\
\text { with statin }\end{array}$ \\
\hline $\begin{array}{l}\text { Alirocumab } \\
\text { (Praluent) }\end{array}$ & $\begin{array}{l}75 \text { mg SC, every } 2 \text { weeks } \\
150 \text { mg SC, every } 2 \text { weeks }\end{array}$ & $\begin{array}{l}45 \% \\
58 \%\end{array}$ \\
\hline $\begin{array}{l}\text { Evolocumab } \\
\text { (Repatha) }\end{array}$ & $\begin{array}{l}140 \text { mg SC, every } 2 \text { weeks } \\
420 \text { mg SC, every } 4 \text { weeks }\end{array}$ & $\begin{array}{l}64 \% \\
58 \%\end{array}$ \\
\hline
\end{tabular}

LDL-C = low-density lipoprotein cholesterol; SC = subcutaneously

\section{STATINS}

Important updates to the 2018 cholesterol guidelines include an emphasis on a risk-benefit discussion between the clinician and patient before beginning statin therapy.

Statins remain the first-line drugs for lowering cholesterol and ASCVD risk reduction. The type of statin and dose depends on the intensity or degree of reduction of LDL-C desired (Table 4).

\section{SUMMARY}

With so much awareness and talk about the importance of cholesterol, many have the false impression that patients are being over treated for it. In fact, a very small percentage of patients take 2 lipid-lowering drugs and much of the patient population at high risk for ASCVD is not under control, especially women. The data are robust that patients at high clinical risk for ASCVD should be treated aggressively. For patients at intermediate risk, calcium scoring and attention to risk-enhancing factors can help stratify ASCVD risk, as well as present an opportunity for discussion and shared decision-making. 
TABLE 4

\section{Statin therapy for reduction of LDL-C}

Intensity of LDL-C reduction

Drug

High $(>50 \%) \quad$ Moderate $(30 \%-49 \%)$

Low $(<30 \%)$

Atorvastatin (Lipitor)

$40 \mathrm{mg} / 80 \mathrm{mg}$, daily

Rosuvastatin (Crestor)

$20 \mathrm{mg} / 40 \mathrm{mg}$, daily

Simvastatin (Zocor)

Pravastatin (Pravachol)

Pitavastatin (Livalo)

Lovastatin (Mevacor, Altoprev)

Fluvastatin XL (Lescol XL)

Fluvastatin (Lescol)

$10 \mathrm{mg} / 20 \mathrm{mg}$, daily

$5 \mathrm{mg} / 10 \mathrm{mg}$, daily

$20 \mathrm{mg} / 40 \mathrm{mg}$, daily

$40 \mathrm{mg} / 80 \mathrm{mg}$, daily

$1 \mathrm{mg}$ to $4 \mathrm{mg}$, daily

$40 \mathrm{mg} / 80 \mathrm{mg}$, daily

$80 \mathrm{mg}$, daily

$40 \mathrm{mg}$, twice daily
$10 \mathrm{mg}$, daily

$10 \mathrm{mg} / 20 \mathrm{mg}$, daily

$20 \mathrm{mg}$, daily

$20 \mathrm{mg} / 40 \mathrm{mg}$, daily

LDL-C = low-density lipoprotein cholesterol

\section{REFERENCES}

1. Benjamin EJ, Muntner P, Alonso A, et al; American Heart Association Council on Epidemiology and Prevention Statistics Committee and Stroke Statistics Subcommittee. Heart disease and stroke statistics-2019 update: a report from the American Heart Association [published correction appears in Circulation 2020; 141(2):e33]. Circulation 2019; 139(10):e56-e528. doi:10.1161/CIR.0000000000000659

2. Mercado C, DeSimone AK, Odom E, Gillespie C, Ayala C, Loustalot F. Prevalence of cholesterol treatment eligibility and medication use among adults-United States, 2005-2012 [published corrections appear in MMWR Morb Mortal Wkly Rep 2015; 64(48):1350 and MMWR Morb Mortal Wkly Rep 2016; 64(52):1409]. MMWR Morb Mortal Wkly Rep 2015; 64(47):1305-1311. doi:10.15585/mmwr.mm6447a1

3. Stone NJ, Robinson JG, Lichtenstein AH, et al; American College of Cardiology/American Heart Association Task Force on Practice Guidelines. 2013 ACC/AHA guideline on the treatment of blood cholesterol to reduce atherosclerotic cardiovascular risk in adults: a report of the American College of Cardiology/American Heart Association Task Force on Practice Guidelines [published corrections appear in J Am Coll Cardiol 2014; 63(25 Pt B):3024-3025 and J Am Coll Cardiol 2015; 66(24):2812]. J Am Coll Cardiol 2014; 63(25 Pt B):2889-2934. doi:10.1016/j. jacc.2013.11.002

4. Grundy SM, Stone NJ, Bailey AL, et al. 2018 AHA/ACC/AACVPR/ AAPA/ABC/ACPM/ADA/AGS/APhA/ASPC/NLA/PCNA guideline on the management of blood cholesterol-Executive summary: a report of the American College of Cardiology/ American Heart Association Task Force on clinical practice guidelines [published correction appears in J Am Coll Cardiol 2019; 73(24):3234-3237]. J Am Coll Cardiol 2019; 73(24):3168-3209. doi:10.1016/j.jacc.2018.11.002

5. Hoy MK, Goldman JD. Fiber intake of the U.S. population: what we eat in America, NHANES 2009-2010. Food Surveys Research Group Dietary Data Brief No. 12. September 2014.

6. Institute of Medicine 2005. Dietary reference intakes for energy, carbohydrate, fiber, fat, fatty acids, cholesterol, protein, and amino acids. Washington, DC: The National Academies Press. https://doi. org/10.17226/10490.
7. Haley M. Livestock, dairy and poultry outlook: December 2019. United States Department of Agriculture Economic Research Services website. https://www.ers.usda.gov/publications/pubdetails/?pubid=95579. December 16, 2019. Accessed April 7, 2020.

8. American Heart Association Recommendations for Physical Activity in Adults and Kids. American Heart Association website. https:// www.heart.org/en/healthy-living/fitness/fitness-basics/aha-recs-forphysical-activity-in-adults. Updated April 18, 2018. Accessed April 8, 2020.

9. Facts \& Statistics Physical Activity. U.S. Department of Health and Human Services website. https://www.hhs.gov/fitness/resourcecenter/facts-and-statistics/index.html. Reviewed January 26, 2017. Accessed April 8, 2020.

10. Aviña-Zubieta JA, Choi HK, Sadatsafavi M, Etminan M, Esdaile JM, Lacaille D. Risk of cardiovascular mortality in patients with rheumatoid arthritis: a meta-analysis of observational studies. Arthritis Rheum 2008; 59(12):1690-1697. doi:10.1002/art.24092

11. Tsimikas S. A test in context: lipoprotein(a)—diagnosis, prognosis, controversies, and emerging therapies. J Am Coll Cardiol 2017; 69(6):692-711. doi:10.1016/j.jacc.2016.11.042

12. Jellinger PS, Handelsman Y, Rosenblit PD, et al. American Association of Clinical Endocrinologists and American College of Endocrinology guidelines for management of dyslipidemia and prevention of cardiovascular disease. Endocr Pract 2017; 23(suppl 2):1-87. doi:10.4158/EP171764.APPGL

13. Chen Q, Wu G, Li C, Qin X, Liu R, Zhang M. Safety of proprotein convertase subtilisin/kexin type 9 monoclonal antibodies in regard to diabetes mellitus: a systematic review and meta-analysis of randomized controlled trials. Am J Cardiovasc Drugs 2019 [published online ahead of print Dec 11, 2019]. doi:10.1007/s40256-019-00386-w.

Correspondence: Leslie Cho, MD, Department of Cardiovascular Medicine, Heart, Vascular, and Thoracic Institute, JB1, Cleveland Clinic, 9500 Euclid Avenue, Cleveland, Ohio 44195; chol@ccf.org 